\title{
Update on the DIII-D ECH system: experiments, gyrotrons, advanced diagnostics, and controls
}

\author{
$\underline{\text { John Lohr }^{1}}{ }^{1}$, Rigoberto Brambila $^{1}$, Mirela Cengher ${ }^{1}$, Yuri Gorelov ${ }^{1}$, William Grosnickle ${ }^{1}$, Charles Moeller ${ }^{1}$, \\ Dan Ponce ${ }^{1}$, Antonio Torrezan ${ }^{1}$, Lawrence Ives ${ }^{2}$, Michael Reed ${ }^{2}$, Monica Blank ${ }^{3}$, Kevin Felch ${ }^{3}$, \\ Claudia Parisuaña ${ }^{4}$, Alexandra LeViness ${ }^{5}$ \\ ${ }^{1}$ General Atomics, San Diego, CA USA, lohr@fusion.gat.com \\ ${ }^{2}$ Calabazas Creek Research, San Mateo, CA, USA \\ ${ }^{3}$ Communications and Power Industries, Palo Alto, CA, USA \\ ${ }^{4}$ Pontificia Universidad Católica del Perú, Lima, Peru \\ ${ }^{5}$ University of Alabama, Tuscaloosa, AL, USA
}

\begin{abstract}
The ECH system on DIII-D is continuing to be upgraded, while simultaneously being operated nearly daily for plasma experiments. The latest major hardware addition is a new $117.5 \mathrm{GHz}$ gyrotron, which generated 1.7 MW for short pulses during factory testing. A new gyrotron control system based on Field Programmable Gate Array (FPGA) technology with very high speed system data acquisition has significantly increased the flexibility and reliability of individual gyrotron operation. We have improved the performance of the fast mirror scanning, both by increasing the scan speeds and by adding new algorithms for controlling the aiming using commands generated by the Plasma Control System (PCS). The system is used for transport studies, ELM control, current profile control, non-inductive current generation, suppression of MHD modes, startup assist, plasma density control, and other applications. A program of protective measures, which has been in place for more than two years, has eliminated damage to hardware and diagnostics caused by overdense operation. Other activities not directly related to fusion research have used the ECH system to test components, study methods for improving production of semiconductor junctions and materials, and test the feasibility of using ground based microwave systems to power satellites into orbit.
\end{abstract}

\section{System Overview}

With the addition of a seventh gyrotron to the six in regular operation, about $4.5 \mathrm{MW}$ injected rf power will be available for experiments. Although the pulse lengths are at present limited administratively to $5 \mathrm{~s}$, compatible with the 6-7 s pulse lengths of DIII-D plasmas, the gyrotrons reach thermal equilibrium after a few seconds, and longer pulses will be available when longer duration DIII-D plasmas are being used. Results of tests of the 117.5 $\mathrm{GHz}$ tube will determine the course to be taken with future system upgrades. One additional upgrade, that has been committed at present, will be the combining of parts from two $1 \mathrm{MW} 110 \mathrm{GHz}$ tubes, that suffered collector failures, into a single gyrotron with an improved collector made from $\mathrm{CuCrZr}$ material. This gyrotron should be available in 2018 and boost the injected rf power to greater than $5 \mathrm{MW}$ from eight gyrotrons. The power supplies for all the gyrotrons in the system employ autotransformer/rectifiers, delivering about $90-100 \mathrm{kV}$ to tetrode modulator/regulators that provide highly regulated vol- tage to the gyrotrons, either two in parallel or singly. The $\mathrm{rf}$ is carried in evacuated circular waveguides with diameter $31.75 \mathrm{~mm}$, propagating the $\mathrm{HE}_{1,1}$ mode about $90 \mathrm{~m}$ to the tokamak with $75-80 \%$ efficiency.

\section{Control System Upgrades}

Previously, the gyrotron output power, including a variety of pre-programmed modulation options, was controlled by waveform generators with good time resolution and reliability, but with limited options beyond four waveform choices. These were simple continuous, and three oscillation waveforms, sinusoidal, triangular, and rectangular. Faults from such events as cathode or body overcurrent, loss of rf generation, or failure to track the commanded waveforms, would result in the termination of operation for the faulted gyrotron for that plasma shot. In cases where two gyrotrons were powered from a single tetrode, a fault in either gyrotron would terminate output from both.

All the DIII-D gyrotrons are now controlled by FPGA based logic, which has greatly increased the flexibility of fault handling, improved fault protection and, in a major improvement, allowed restart of the gyrotrons after a fault has cleared. As an example, Fig. 1 shows a case in which a gyrotron faulted, shut off, and then successfully restarted and continued generating full power as if nothing had happened. The FPGA controller is programmed to look for inconsistencies between the applied voltages and the measured performance parameters, such as output rf power, body current and cathode current, all digitized at $2 \mathrm{MS} / \mathrm{s}$. In this case, the system detected a very small oscillation of the body current when the body voltage was stable. This inconsistency triggered a rapid shutdown of the gyrotron within about $50 \mu \mathrm{s}$ and a rapid restart to full output within about $50 \mathrm{~ms}$. The brief fault was not a factor for the experiment and the shot, which previously would have been a failure, was in practice a success.

This capability has also allowed a modest increase in delivered rf power, since the gyrotrons can now be operated closer to the point where higher power is achieved but at risk of loss of rf generation. The tubes will now be restarted in this event.

\section{ELM Control with ECCD}

After Edge Localized Modes (ELMs) were recognized as a potentially serious threat to the safe operation 


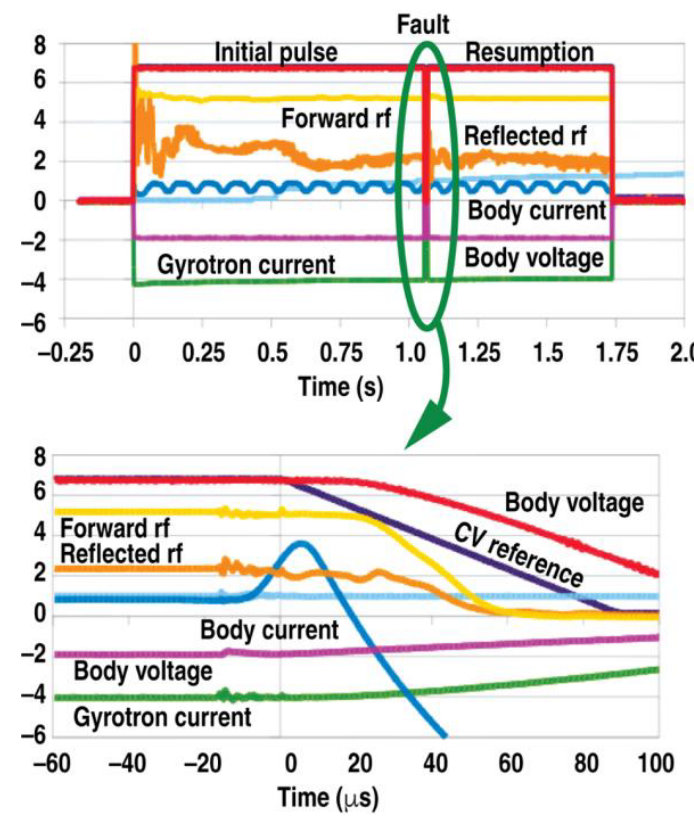

Fig. 1. In this case, an incipient body current fault was identified by the FPGA controller. Rapidly halting the shot enabled an immediate restart with nearly no lost time

of large devices such as ITER, research programs have attempted both to understand these fluctuations and to mitigate their effects. On the front lines of this effort are ECH/ECCD. The microwave systems have been used in conjunction with externally applied 3D fields to suppress

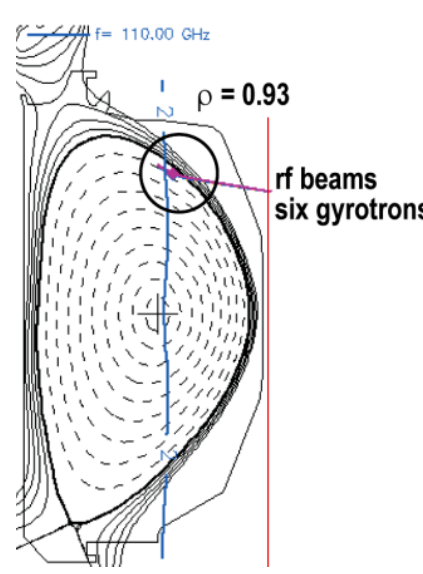

or augment ELMs in studies of the ELM phenomenon. An example is shown in Fig. 2, in which ECCD in the plasma periphery from six gyrotrons, generating current in the direction opposite to the plasma current, successfully suppressed ELMs. A large number of similar experiments have studied the ability of $\mathrm{ECH} / \mathrm{ECCD}$ to mitigate this instability.
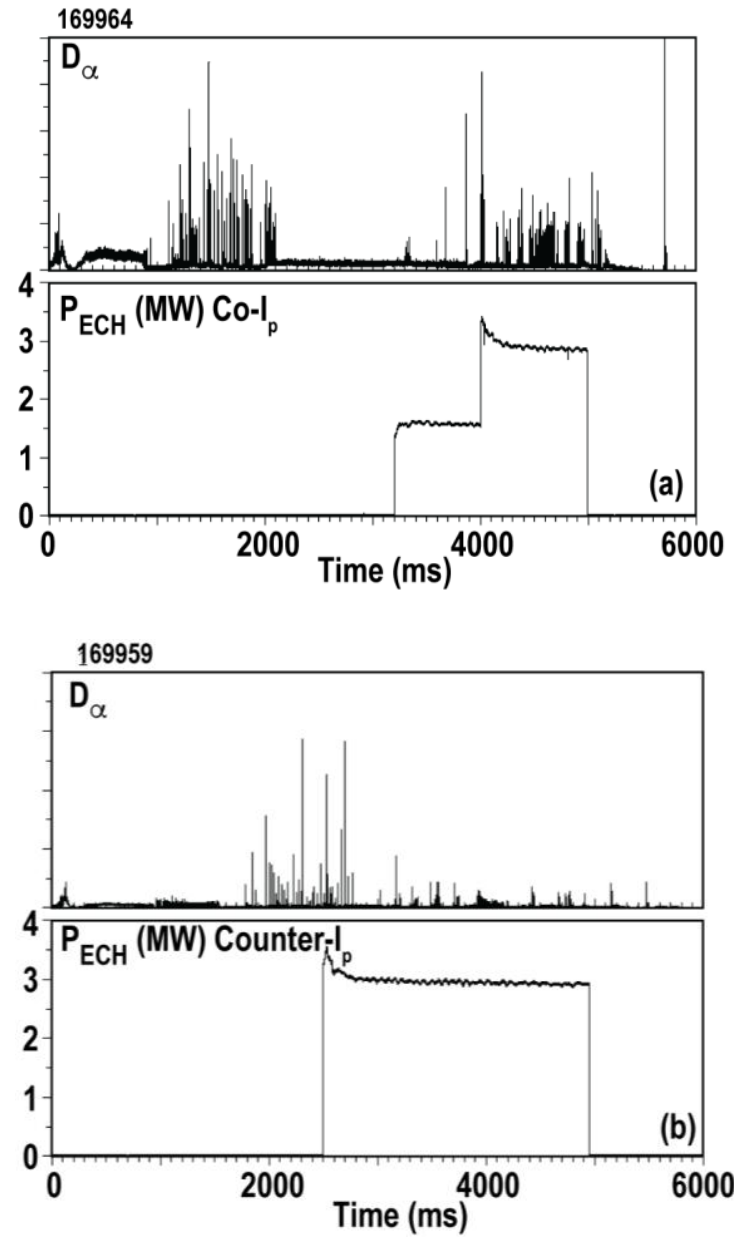

Fig. 2. This example shows the effect on ELMs of about $3 \mathrm{MW}$ of electron cyclotron power deposited in the edge of the plasma beyond rho $=0.9$. The small amount of EC driven current was opposite to the plasma current for suppression and in the direction of the plasma current for augmentation of the ELMs. Different combinations of externally applied magnetic fields can reverse the effect of the ECH

\section{Acknowledgment}

This work was funded by the U.S. DOE under Cooperative Agreement DE-FC02-04ER54698. DIII-D data shown in this paper can be obtained in digital format by following the links

at https://fusion.gat.com/global/D3D_DMP. 